Journal of Modern Optics

Vol. 00, No. 00, 00 Month 200x, 1-20

\title{
Measurement-driven dynamics for a coherently-excited atom
}

\author{
Andrew J.T. Colin ${ }^{a *}$ Stephen M. Barnett ${ }^{a}$ and John Jeffers ${ }^{a}$ \\ ${ }^{a}$ Department of Physics, University of Strathclyde, John Anderson Building, Glasgow G4 0NG, UK; \\ (v1.0 released October 2012)
}

The phenomenon of telegraphing in a measurement-driven two-level atom was noted in [1]. Here we introduce two quantitative measures of telegraphing: one based on the accumulated measurement record and one on the evolution of the quantum state. We use these to analyse the dynamics of the atom over a wide range of parameters. We find, in particular, that the measures provide broadly similar statistics when the measurements are frequent, but differ widely when measurements are sparse. This is in line with intuition, and demonstrates the utility of both measures.

Keywords: Master equations; Lindblad form; Markov processes; Telegraphing; Rabi cycle; Two-state atoms; Imperfect measurements 


\section{Introduction}

The idea of random telegraph dynamics for a single quantum system started with the proposal by Dehmelt [2] to detect a weak transition by monitoring the fluorescence on a strong one, as depicted in Figure 1 . The fluorescence observed on the strong transition is interrupted by periods of darkness during which, we can infer, an absorption has occurred on the weak transition [3, 4]. Today, the observation of such quantum jumps is a standard technique for counting trapped ions [5].

In this paper we investigate the effect of a sequence of measurements on the otherwise coherent dynamics of a driven two-level atom. On average the dynamics of an ensemble of such atoms exhibit smooth but damped Rabi oscillations, but individual atoms display a spectrum of behaviour ranging from interrupted Rabi oscillations to a random telegraph [1]. Central to our approach is the quantum Monte Carlo method [6-9] in which we simulate the evolution of a driven single two-level atom interrupted by instantaneous measurements applied stochastically to the atom. This gives us access to both the evolution of the state and also to the associated measurement record.

A closed quantum system is one that is free to evolve in time without any external influence. Such systems obey Schrődinger's equation; their evolution is predictable and - in principle reversible. In practice, closed quantum systems do not occur in nature (except, arguably, for the whole universe). The best that can be done experimentally is to isolate a system for a very short time.

Imperfect measurement leads to a possible method of analysing quantum systems which are open, and do not evolve according to Schrődinger's equation. A more general form for evolution of the reduced density operator of an open system is a master equation in the Lindblad form, which assumes that the evolution is Markovian, and that the environment of the system is large enough not to be changed by the process [10-12]:

$$
\frac{d \hat{\rho}}{d t}=\frac{-i}{\hbar}[\hat{H}, \hat{\rho}]+\sum_{j} \gamma_{j}\left(\hat{K}_{j} \hat{\rho} \hat{K}_{j}^{\dagger}-\frac{1}{2} \hat{K}_{j}^{\dagger} \hat{K}_{j} \hat{\rho}-\frac{1}{2} \hat{\rho} \hat{K}_{j}^{\dagger} \hat{K}_{j}\right),
$$

where $\hat{\rho}$ is the reduced density operator, and $\hat{H}$ the system hamiltonian. The first term of the equation expresses its closed evolution, which would take place if there were no external effects. $\gamma$ is a rate parameter; $K_{j}$, are called Kraus operators [13], and depend on the manner and strength of the external influences on the system. The Lindblad form has been successfully used in several contexts, including friction [14, 15].

It has been known since the publication of Von Neumann's book [16] in 1927, that a measurement of a quantum system will transform it to one of its eigenvalues, overriding Schrödinger's equation. To be specific, if the density operator of a quantum system at time $t$ is

$$
\hat{\rho}_{t}=\left(\begin{array}{ll}
a_{t} & x_{t} \\
y_{t} & b_{t}
\end{array}\right)
$$

then a measurement will ideally return ' 1 ' with probability $a_{t}$, and ' 0 ' with probability $b_{t}$, leaving the system in the state that corresponds to the measurement. More recently, it was realised 


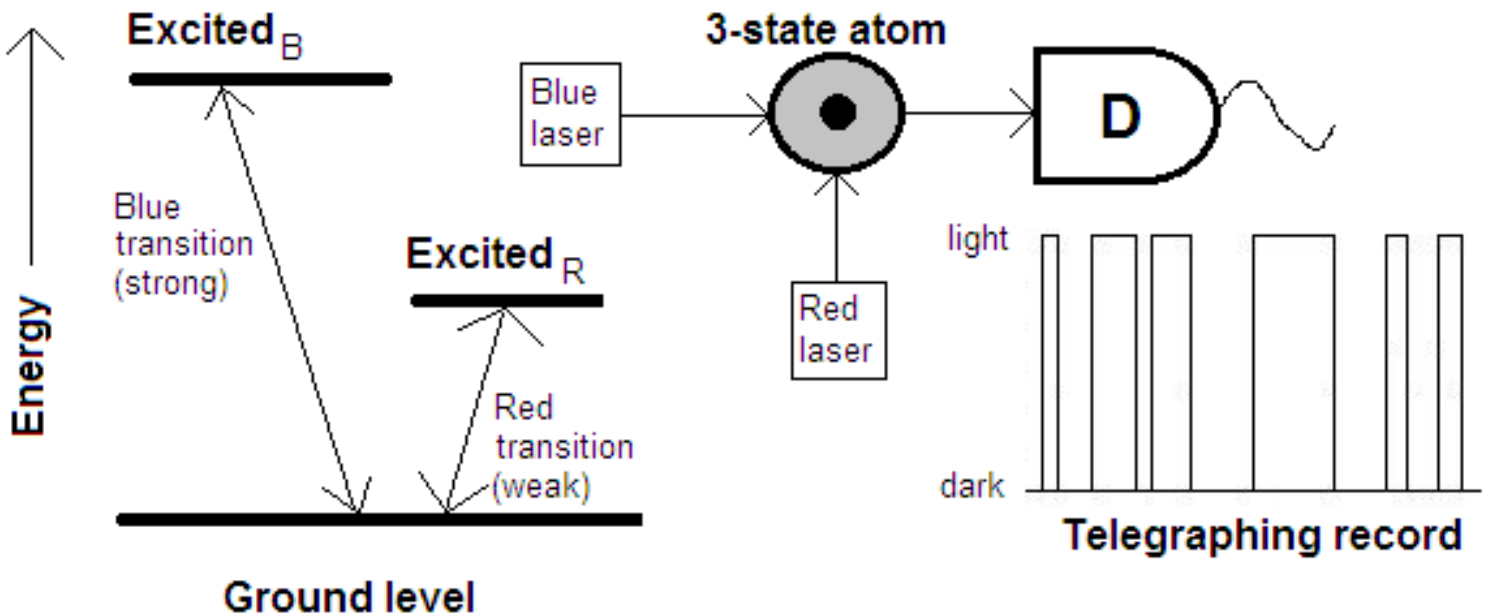

Figure 1. States of a three-state atom

that a measurement might depart from the ideal and give a result not always in accordance with the probability amplitudes. Consider a defective measurement device, which only returns the right state with probability $p(p \leq 1)$. When a measurement made with this device delivers the result ' 1 ' (which nominally corresponds to state $|1\rangle$ ), it actually measures the system to have been in the state $p|1\rangle\langle 1|+(1-p)| 0\rangle\langle 0|$. Given a state with the density operator $(2)$ the measurement will return ' 1 ' with probability $p a_{t}+(1-p) b_{t}$. The state of the system after such a measurement depends upon the details of the measurement interaction. Unless $p=1$ (for a von Neumann measurement) the resulting state will not be one of its eigenstates but something intermediate. In particular, if $p$ is near to 0.5 , the measurement yields very little information.

As imperfect measurements can induce changes in a system (just like external influences that gradually destroy its state) it might be possible to use a stream of continuous imperfect measurements as a surrogate for external influences, with the advantage that such a stream is easier to analyse than random external effects. This idea was first taken up in papers by Hornburger [17] and Cresser, Barnett, Jeffers and Pegg [1], and further developed by Everitt, Munro and Spiller [18]. The subject of the present paper is the quantitative analysis of a stream of imperfect measurements on a quantum system.

\section{Background}

This section is a summary of the paper Measurement master equation, [1], which forms the background to our own investigation.

The authors derived and used a master equation $[10-12,19,20]$ to examine the behaviour of a quantum system under imperfect, but frequent measurement. The physical system they considered is an isolated two-state atom, with the bare energy eigenstates $|1\rangle$ and $|0\rangle$, resonantly driven by an electromagnetic field. On its own, this dressed atom forms a closed system. The probable state of the atom undergoes Rabi oscillations, at a rate that depends on the strength of the applied field.

It is helpful to treat the atomic state as effectively spinning, and in this context we introduce 
two Pauli operators:

$$
\begin{gathered}
\hat{\sigma}_{1}=|0\rangle\langle 1|+| 1\rangle\langle 0|, \\
\hat{\sigma}_{3}=|1\rangle\langle 1|-| 0\rangle\langle 0| .
\end{gathered}
$$

Then the hamiltonian[10] that describes the interaction between the field and the atom is

$$
\hat{H}=-\frac{\hbar \Omega}{2} \hat{\sigma}_{1}
$$

where $\Omega$ is the Rabi frequency.

The application of measurements implies that the system is no longer closed. The evolution of the atomic density operator may be described by a master equation of the form given in the introduction.

Energy measurements would ideally correspond to the projectors $|1\rangle\langle 1|$ and $|0\rangle\langle 0|$. However, the measurements may be imperfect, and have only a probability $p$ of correctly idntifying the state of an atom prepared in the state $|1\rangle$ or $|0\rangle$. This is then a generalised measurement described by probability operator measure ('POM') with the elements:

$$
\begin{gathered}
\hat{\pi}_{1}=p|0\rangle\langle 0|+(1-p)| 1\rangle\langle 1|, \\
\hat{\pi}_{0}=p|1\rangle\langle 1|+(1-p)| 0\rangle\langle 0| .
\end{gathered}
$$

These operators are both hermitian and positive, and they satisfy the requirement that $\sum_{j} \hat{\pi}_{j}=$ 1 (where $\mathbf{1}$ is the identity operator).

As the interaction with the environment takes the form of measurement, each pair of Kraus operators corresponds to one of the elements of the POM, according to the rule

$$
\hat{\pi}_{j}=\hat{K}_{j}^{\dagger} \hat{K}_{j}
$$

These relationships do not specify a unique set of operators $K_{j}$. The simplest realisations of these Kraus operators are the square roots of the POM elements which, for our two probability operators, are:

$$
\begin{aligned}
& \hat{K}_{1}=\hat{K}_{1}^{\dagger}=\sqrt{p}|1\rangle\langle 1|+\sqrt{1-p}| 0\rangle\langle 0| \\
& \hat{K}_{0}=\hat{K}_{0}^{\dagger}=\sqrt{p}|0\rangle\langle 0|+\sqrt{1-p}| 1\rangle\langle 1|
\end{aligned}
$$

Having defined the concept of a imperfect measurement, the paper then considers the aspect of measurement frequency. Measurements occur at random times, and the authors use a parameter $R$, which indicates the average number of measurements per unit time. The presence of $R$ modifies the basic master equation, as follows:

$$
\mathcal{T}(\hat{\rho})=\frac{-i}{\hbar}[\hat{H}, \hat{\rho}]+R\left[\sum_{j}\left(\hat{K}_{j} \hat{\rho} \hat{K}_{j}^{\dagger}-\frac{1}{2} \hat{K}_{j}^{\dagger} \hat{K}_{j} \hat{\rho}-\frac{1}{2} \hat{\rho} \hat{K}_{j}^{\dagger} \hat{K}_{j}\right)\right]
$$


The fact that

$$
\sum_{j}\left(\hat{K}_{j}^{\dagger} \hat{K}_{j}\right)=\mathbf{1}
$$

enables us to write the master equation as:

$$
\frac{d \hat{\rho}}{d t}=\left(\frac{i \Omega}{2}\right)\left[\hat{\sigma}_{1}, \hat{\rho}\right]+\gamma\left(\hat{\sigma}_{3} \hat{\rho} \hat{\sigma}_{3}-\hat{\rho}\right),
$$

where

$$
\gamma=\frac{R}{2}(\sqrt{1-p}-\sqrt{p})^{2} .
$$

The analytic solution of this equation shows that starting in a pure state $(|\psi\rangle=|0\rangle$ or $|\psi\rangle=|1\rangle$ a sufficiently large ensemble of atoms will decay into an average mixed state

$$
\hat{\rho}=\frac{1}{2}(|0\rangle\langle 0|+| 1\rangle\langle 1|) .
$$

The decay is oscillatory if $\gamma$ is small, but exponential for larger values of $\gamma$.

The evolution can also be solved numerically using Monte Carlo methods to give individual quantum trajectories for the atom. The intervals between measurements depend on a random number generator and the statistics of the measurements are provided by a random process which mimics the measurement statistics associated with the state. These trajectories are quite different from the analytic solution; they do not decay, and with high values of $\gamma$ they exhibit telegraphing, where the Rabi cycle is suppressed and the atom spends long periods in or near the upper or lower energy level. Figures 2 to 5 show some simulated trajectories. Figure 2 uses a very low value of $\gamma$. The basic Rabi cycle is hardly disturbed and continues indefinitely. Figure 3 is a trajectory produced by an intermediate value of $\gamma$, and it is not obvious, from the graph, whether telegraphing is occurring or not. Figure 4 uses a much higher value of $\gamma$, and demonstrates the telegraphing effect. The horizontal scale has been extended to 20 Rabi cycles. Figure 5 illustrates the details of a trajectory when the system is telegraphing, and switches from one state to the other. The 'spikes' can only occur when the measurements are imperfect.

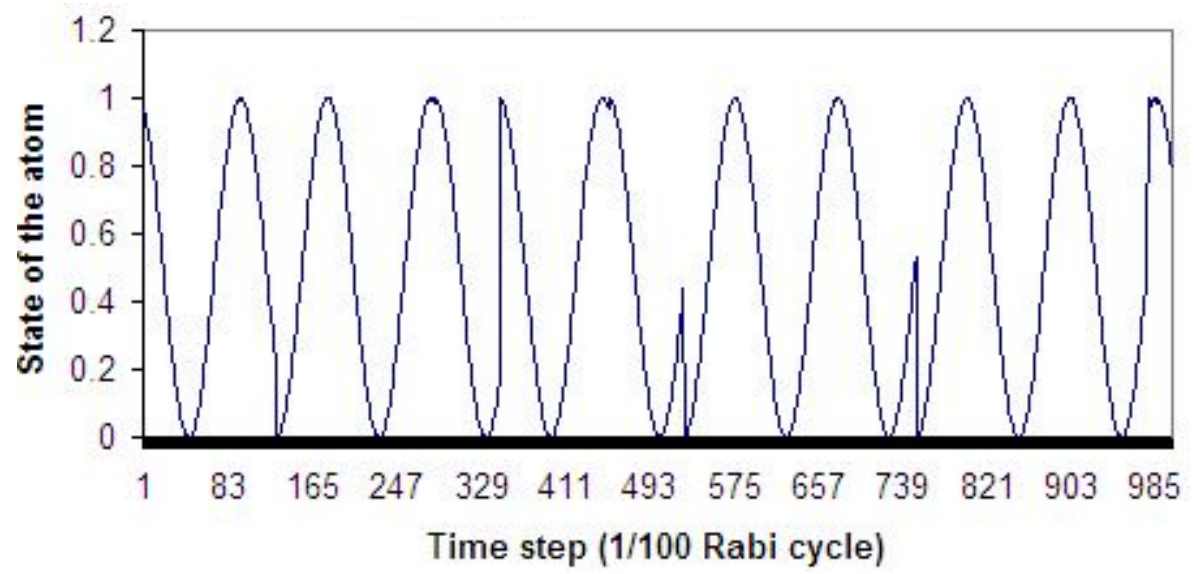

Figure 2. Trajectory with $p=1$ and $\gamma=0.005$ 


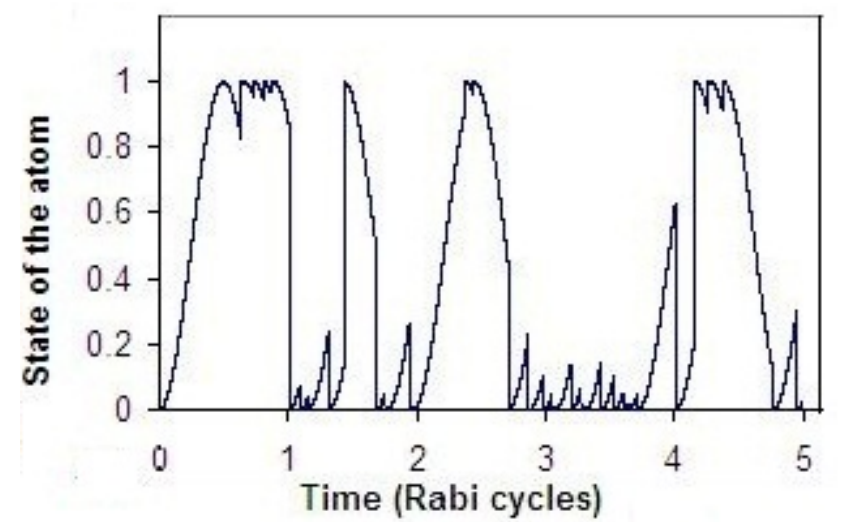

Figure 3. Trajectory with $p=1$ and $\gamma=0.04 \mathrm{a}$

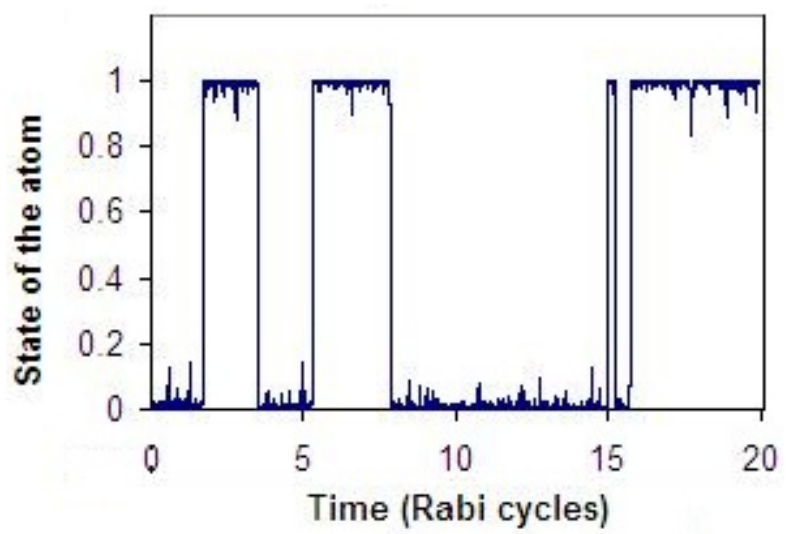

Figure 4. Trajectory with $p=1$ and $\gamma=0.25$

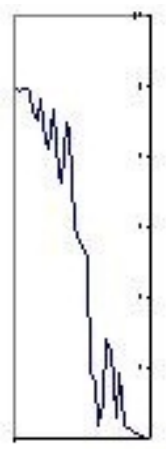

Figure 5. Typical switching trajectory when $p<1$

In the final section of their paper, the authors provide a qualitative description of several phenomena which can be seen in these trajectories. In brief, low values of $\gamma$ have little effect on the continuous Rabi cycle. High values, combined with near-perfect measurement, produce telegraphing. Each dwell at one of the eigenstates is 'whiskery' because every measurement is followed by a short period during which the atom executes a fraction of a Rabi cycle, before being forced back to the eigenstate. When a measurement is imperfect, it can leave the atom in an intermediate state. This may lead to 'jagged' state changes, as shown in figure 5 . 


\section{A quantitative analysis}

Here we offer a quantitative analysis of the phenomena described in [1].

\subsection{The simulator}

Our results are derived from a simulator, which is based on a 4th-order Runge Kutta integration procedure using a time-step, $\delta t$, of $\frac{1}{1000}$ of a Rabi cycle. Our reported statistics are based on runs of 1000 Rabi cycles, or $10^{6}$ time-steps. The simulator takes two parameters:

- $R$, the average rate of measurements. In the simulator this is converted to a fraction $q$, the probability of a measurement in any one time-step.

$$
q=\delta t\left(\frac{2 \pi R}{\Omega}\right)
$$

where $\Omega$ is the angular frequency of the Rabi cycle.

- $p$, the probability that a measurement returns a result in keeping with the current value of the density operator. There is a probability $(1-p)$ that this result be inverted.

The Rabi cycle is simulated by solving Schrődinger's equation

$$
\frac{d \hat{\rho}}{d t}=\frac{-i}{\hbar}[\hat{H}, \hat{\rho}]
$$

where

$$
\hat{H}=\left(\begin{array}{cc}
0 & \frac{-\hbar \Omega}{2} \\
\frac{-\hbar \Omega}{2} & 0
\end{array}\right)
$$

and $\Omega$ is the angular frequency of the Rabi oscillation.

As an initial value we take

$$
\hat{\rho}=\left(\begin{array}{ll}
1 & 0 \\
0 & 0
\end{array}\right)
$$

which corresponds to an initially excited atom.

The occurrence of a measurement during any time cycle is determined by a rectangular random number generator with a range $(0 \leq g \leq 1)$. A measurement takes place if $(g<q)$. It is assumed that measurements are so rare that there will never be more than one measurement in the same time-step. The validity of the model is bounded in two ways:

- The model is inaccurate for very high values of $R$, as there is then a significant chance that two or more measurements could fall in the same time-step. The simulator does not handle this situation, but its validity could be extended by using a shorter time-step.

- When $R$ is very low, there are not enough events, even during 1000 Rabi cycles, to yield accurate statistics. A longer period of observation would mitigate this problem.

Notwithstanding these practical limitations, the model is accurate over a wide range of values for $R:(0.01 \leq R \leq 1000)$. 
The overall effect of an imperfect measurement was computed as follows: suppose that the atom is part-way through its Rabi cycle, so that the probability of its being in state $|1\rangle$ is $x$. Then an ideal measurement, using the projectors $|1\rangle\langle 1|$ and $|0\rangle\langle 0|$, would return $|1\rangle$ with probability $x$, and $|0\rangle$ with probability $(1-x)$. But the defective measurement only records the ideal result with probability $p$, so the actual probabilities of results ' 1 ' and ' 0 ' are

$$
\begin{gathered}
P(1)=p x+(1-p)(1-x), \\
P(0)=p(1-x)+(1-p) x .
\end{gathered}
$$

If the measurement is imperfect, the final state of the atom will not be the same as the result of the measurement $[21,22]$. If the measurement returns result $j$ the change in the density operator is

$$
\hat{\rho} \rightarrow \frac{\hat{K}_{j} \hat{\rho} \hat{K}_{j}^{\dagger}}{\operatorname{Tr}\left(\hat{K}_{j} \hat{\rho} \hat{K}_{j}^{\dagger}\right)},
$$

where $K_{j}$ and $K_{j}^{\dagger}$ are defined above, in (8). If $p=1$ the effect is to shift the density operator exactly to one of the states $|0\rangle$ or $|1\rangle$, but for any other value of $p$ the density operator is moved towards one of the states without actually reaching it.

\subsection{Definitions of telegraphing}

The atom under investigation alternates between two phases which we term 'up' dwells, associated with the eigenstate $|1\rangle$, and 'down' dwells, associated with $|0\rangle$. Measurements which move the state of the atom towards a given state are 'up' or 'down' measurements, respectively.

Telegraphing can be defined in two different ways:

- The Measurement Centred method relies entirely on the record of measurements. A dwell is defined as the interval between the first measurement to yield a given result (say ' 1 ') and the next following measurement that gives the opposite result. Intermediate measurements that give the same result are ignored. If measurements are widely spaced the atom may undergo several Rabi oscillations during the dwell. If there are no measurements, the dwell time is infinite. This model has the advantage that the statistics rely entirely on the outcomes of measurements, which implies that they could be obtained from real (not simulated) systems.

- The State Centred method defines a dwell as the uninterrupted time period during which the probability of the atom being at or near one of its energy states $|0\rangle$ or $|1\rangle$ is greater than 0.5. This is not observable, and can only be found by a device or simulator that 'knows' or can estimate the state of the system between measurements. On the other hand, this definition is closer to the intuitive understanding of telegraphing, especially for high values of $\gamma$.

When an atom undergoes an undisturbed Rabi evolution without any measurements, then, for the state-centred method, there will be two dwells for each Rabi cycle. The statecentred method forms a base for the telegraph index, a numerical descriptor of the behaviour of an atom. Figure 4 illustrates a trajectory with state-centred dwells, most of which last considerably longer than one Rabi cycle. The trajectory shows random-telegraph dynamics.

We present theoretical analyses for both methods with perfect measurement, and compare the results with readings taken from the simulator. 


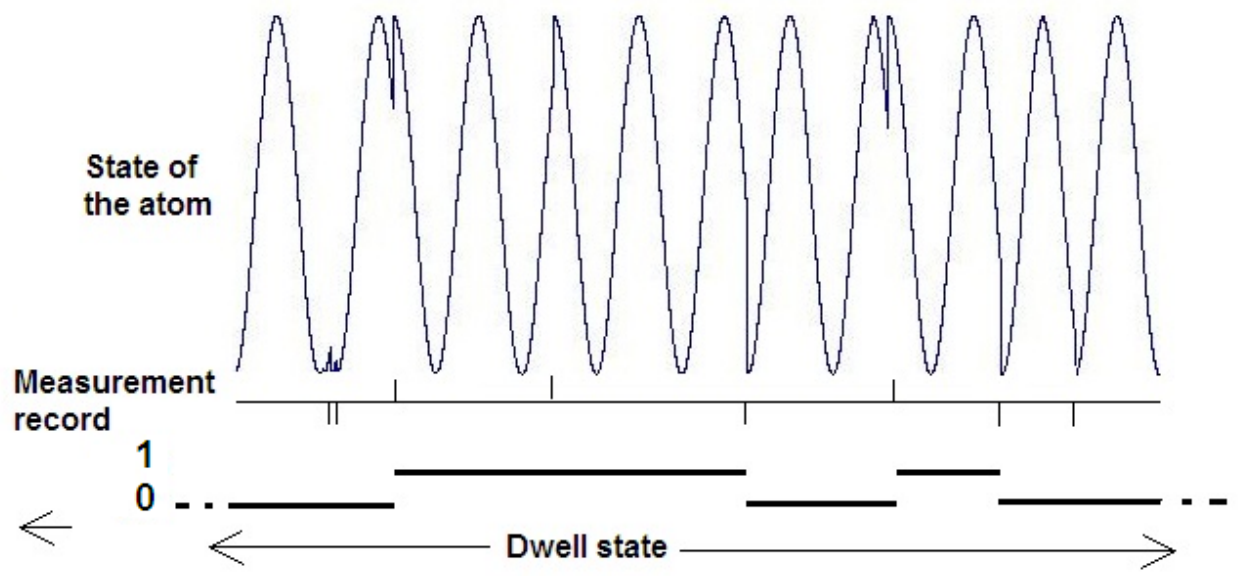

Figure 6. A typical measurement-centred dwell record with $p=1$

\subsection{Telegraph Index}

A comparison of figures 4 and 2 clearly shows that telegraphing is present in one case, but not in the other. Figure 3 cannot readily be assigned to either of these classes; the presence of telegraphing is not a binary quality. This suggests the definition of a 'telegraphing measure' or index $T$, which would vary between 0 when a system is not telegraphing at all, and 1 when measurement is so frequent that the system almost never changes state.

We define the telegraph index to be the time-averaged quantity:

$$
T=\frac{1}{\tau} \int_{0}^{\tau} d t\left[2\left\langle\hat{\sigma}_{3}\right\rangle^{2}-1\right]
$$

where $\tau$ greatly exceeds the Rabi period. For pure Rabi oscillations this quantity is zero, but for a perfect telegraph evolution, in which at all times the atom is in state $|0\rangle$ or state $|1\rangle$, it will be unity. Applying this formula to the trajectories in figures 2 to 4 we find:

\begin{tabular}{|c|c|}
\hline Figure & Telegraph Index \\
\hline Figure 2 & 0.019 \\
Figure 3 & 0.672 \\
Figure 4 & 0.958 \\
\hline
\end{tabular}

We would expect the telegraph index to be asymptotic to 1 as $\gamma$ increases indefinitely. Figure 7, which includes the points listed above and some others, shows that this is so. The fit is not exact, as the points on the graph are derived from the simulator which (of necessity) uses random elements.

\subsection{Analysis of the measurement centred method, with perfect measurements}

This analysis is based on [20]. In this section we assume that measurements that give correct results $(p=1)$. A jump is a measurement result that is different to that of the preceding measurement, and a no-jump is a measurement that gives the same result. Both types of measurement result leave the atom in one or other of the states $|0\rangle$ or $|1\rangle$.

If $\Omega$ is the Rabi frequency, then the uninterrupted Rabi evolution gives

$$
|\psi\rangle=\cos \left(\frac{\Omega t}{2}\right)|1\rangle+i \sin \left(\frac{\Omega t}{2}\right)|0\rangle .
$$




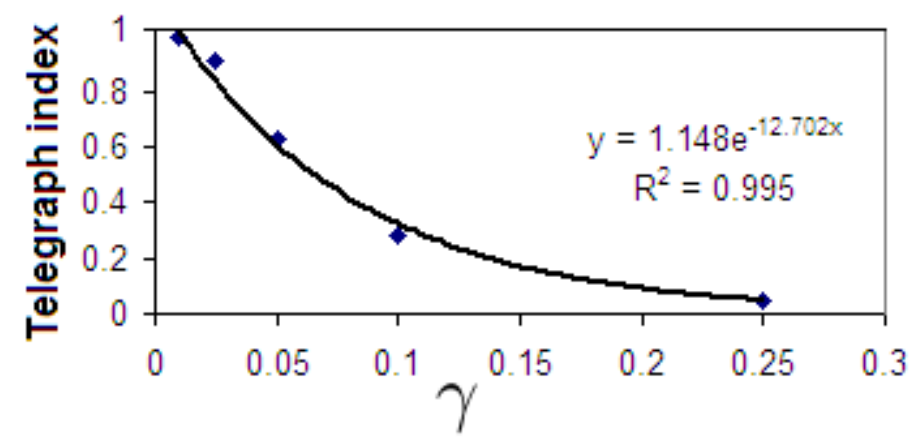

Figure 7. Plot of ( 1 - Telegraph Index $)$ vs. $\gamma$, with exponential fit

The first measurement after $t=0$ occurs between $t$ and $t+d t$. The probability $P_{1}$ of a jump is:

$$
P_{1}=e^{-R t} R d t \sin ^{2}\left(\frac{\Omega t}{2}\right)
$$

Likewise, the probability $\sim P_{1}$ of a no-jump is

$$
\sim P_{1}=\left(1-P_{1}\right)=e^{-R t} R d t \cos ^{2}\left(\frac{\Omega t}{2}\right)
$$

A dwell includes any number of no-jumps (including none), and is terminated by a single jump. We can now extend this to the case of several 'no-jumps', at times $t^{\prime}, t^{\prime \prime}, \cdots$ (working backwards) followed by a jump at time $t$. For example,

$$
P_{3}=e^{-R t} R^{3} d t^{\prime \prime} d t^{\prime} d t \sin ^{2}\left(\frac{\Omega\left(t-t^{\prime}\right)}{2}\right) \times \cos ^{2}\left(\frac{\Omega\left(t^{\prime}-t^{\prime \prime}\right)}{2}\right) \cos ^{2}\left(\frac{\Omega t^{\prime \prime}}{2}\right) .
$$

The average time $\bar{T}_{m}$ between changes of the measured state is then:

$$
\begin{aligned}
\bar{T}_{m}= & \int_{0}^{\infty} d t t\left(P_{1}+P_{2}+P_{3}+\ldots\right) \\
= & \int_{0}^{\infty} R t e^{-R t} d t\left[\sin ^{2}\left(\frac{\Omega t}{2}\right)\right. \\
& +\int_{0}^{t} R d t^{\prime} \sin ^{2}\left(\frac{\Omega\left(t-t^{\prime}\right)}{2}\right) \cos ^{2}\left(\frac{\Omega t^{\prime}}{2}\right) \\
& +\int_{0}^{t} R d t^{\prime \prime} \sin ^{2}\left(\frac{\Omega\left(t-t^{\prime}\right)}{2}\right) \cos ^{2}\left(\frac{\Omega\left(t^{\prime}-t^{\prime \prime}\right)}{2}\right) \cos ^{2}\left(\frac{\Omega t^{\prime \prime}}{2}\right) \\
& +\ldots]
\end{aligned}
$$

Differentiating under the integral sign, and converting to Laplace transforms, we obtain 


$$
\begin{aligned}
& S=\mathcal{L}\left[\int_{0}^{\infty} e^{-q R t} d t \sin ^{2}\left(\frac{\Omega t}{2}\right)\right]=\frac{1}{2}\left[\frac{1}{q R}-\frac{q R}{q^{2} R^{2}+\Omega^{2}}\right], \\
& C=\mathcal{L}\left[\int_{0}^{\infty} e^{-q R t} d t \cos ^{2}\left(\frac{\Omega t}{2}\right)\right]=\frac{1}{2}\left[\frac{1}{q R}+\frac{q R}{q^{2} R^{2}+\Omega^{2}}\right] .
\end{aligned}
$$

It then follows that

$$
\bar{T}_{m}=\left.\left(\frac{-d}{d q}\right)\left[S(q R)+R C(q R) S(q r)+R^{2} C^{2}(q R) S(q R)+\cdots\right]\right|_{q=1}
$$

Differentiating and simplifying, we find

$$
\bar{T}_{m}=\frac{2 R}{\Omega^{2}}+\frac{2}{R} .
$$

This is the dwell time, or average time between changes of state. Using similar methods, we can also find $\overline{S D}$, the standard deviation of the dwell times:

$$
\overline{S D}=\sqrt{\left(\frac{4 R^{2}}{\Omega^{4}}+\frac{4}{R^{2}}\right)} .
$$

A more detailed version of this analysis can be found in [23].

\subsubsection{Statistics of measurement-centred dwells}

Measurements on the simulator are subject to the expected statistical variation, but the averages show excellent agreement with the dwell lengths predicted by our formula. We ran the simulator for some 60 values of $\mathrm{R}$ ranging from 0.01 to 1000 measurements per Rabi cycle, increasing exponentially in steps of 1.2 . Figure 8 is a plot of $\log R$ against $\log \bar{T}_{m}$; we use $\operatorname{logs}$ as otherwise small values of $R$ and $T_{m}$ would disappear.

The calculated and measured standard deviations also agree well, and yield a plot almost identical to Figure 8. For any value of $R$, the average dwell time is about the same as its standard deviation, which confirms that the dwell times have a negative exponential distribution.

The corresponding analysis for imperfect measurements is beyond the scope of this paper, but when measurements are sufficiently far apart to ensure that a random number of Rabi cycles elapse between one measurement and the next, both outcomes of the later measurement are equally likely. This is not changed by a fractional success rate. We conclude that for sparse measurements the measurement-centred dwell time is independent of the accuracy of the measurements.

\subsection{Telegraphing statistics for the state centered method with perfect measurement}

Any perfect measurement of the atom will move its state to exactly one or other of the states $|0\rangle$ or $|1\rangle$ This fact simplifies the analysis. When an atom is telegraphing, the state-centred 


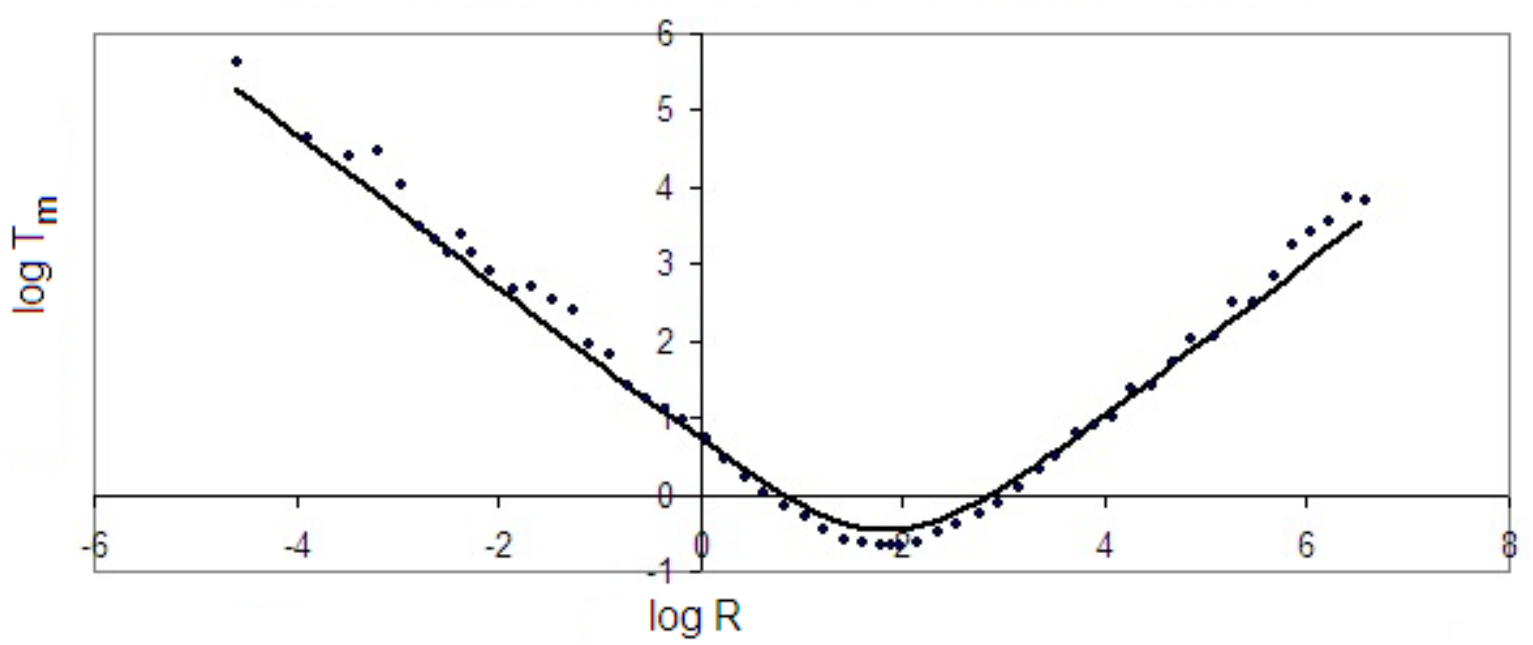

Figure 8. Agreement between calculated and simulated dwell times for Measurement-centred method with $p=1$. The line is calculated, and the points are read from the simulator.

method assumes that it dwells at or near one of its two possible energy eigenstates for extended periods before it switches to the other. In this section we calculate the statistics of dwells for a range of values of the parameter $R$, the mean measurement frequency, and compare the results with measurements made on actual (simulated) trajectories. The heart of the method consists of computing the average evolution of the system for one 'dwell' which consists of one or more 'episodes'. Our explanation is based on an 'up' dwell near $|1\rangle$, the high-energy state, but the problem is symmetrical about the two states, and we could equally well have chosen the lowenergy state.

The analysis of the state-centred method is complicated because there are two different ways that an atom can change its state. On one hand, a measurement that returns the opposite value to the current dwell state causes an immediate switch, but on the other hand, in the absence of measurements, the natural Rabi evolution will bring about a switch in a quarter of a Rabi cycle, as the atom passes through the half-way state - that is, the state in which a measurement would be equally likely to show that a transition had occurred, or that it had not.

In many cases, an 'up' dwell starts in the $|1\rangle$ state, directly following an 'up' measurement in the preceding 'down' dwell. However, the preceding dwell might have ended with a quarter Rabi cycle without measurements, in which case the dwell will start in the half-way state. A way to resolve this complexity is to consider the dwell as consisting of two components:

An initial segment that starts in the half-way state, followed by a final segment that starts in one of the eigenstates - say $|1\rangle$. In many cases the initial segment will be absent, and in some cases the final segment will vanish. Figure 9 illustrates two types of final segment, and figure 10 shows three types of initial segment.

(1) Type 1 final segments start with an 'up' measurement from the preceding 'down' dwell. As time passes, the atom begins to Rabi cycle, until another measurement occurs. As the state is still near $|1\rangle$, the probable result is another 'up' measurement which restores the atom exactly to the $|1\rangle$ state. The exact probability depends on how far the atom has progressed on its Rabi cycle since the last measurement. This continues until a measurement returns 'down' . This ends the dwell and starts the following 'down' dwell at its eigenvalue, without an initial segment.

(2) Type 2 final segments start in the same way, but ends when the gap between measurements is quarter of a Rabi cycle, which is long enough for the atom to reach the state where the 


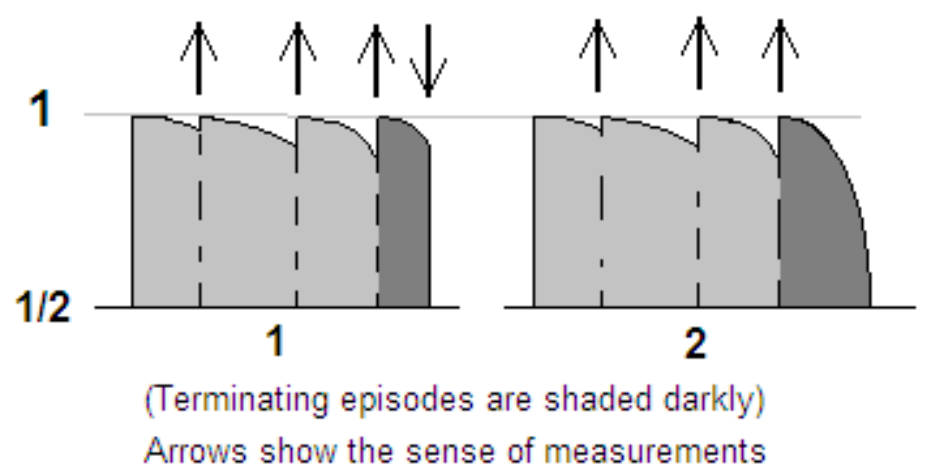

Figure 9. Final segment types (with episodes)

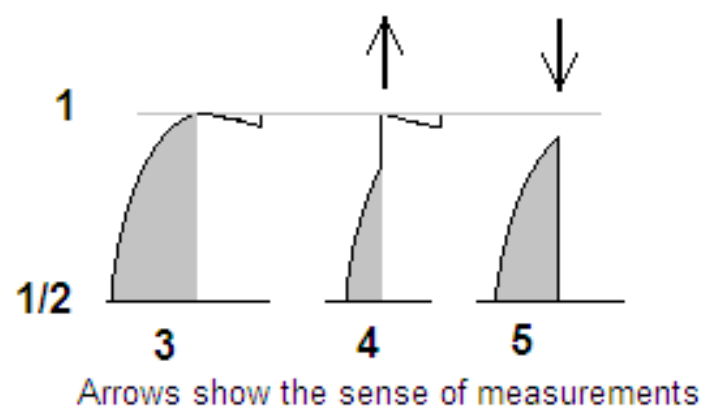

Figure 10. Initial segment types

two eigenstates are equally probable. This ends the current dwell and starts the next one, with an initial segment that starts in the half-way state.

(3) Type 3 initial segments start in the half-way state, when the preceding 'down' dwell ends with a quarter Rabi cycle. The initial quarter Rabi cycle is undisturbed by a measurement.

(4) Type 4 initial segments start in the half-way state, but are interrupted by 'up' measurements.

(5) Type 5 initial segments start in the half-way state, but are interrupted by 'down' measurements. This type of segment is not followed by a final segment, but leads directly to a dwell in the opposite state.

Now we introduce episodes, the individual components that constitute a segment. An episode starts when the system is exactly in the upper energy eigenstate, at time $t=0$, and will evolve away from that state. The episode can end in any of three ways:

(1) A measurement can return it to the initial eigenstate (the dwell continues)

(2) A measurement can switch it to the other eigenstate (the dwell ends, and the next one starts at the eigenvalue in the opposite phase)

(3) If no measurements occur, the atom will execute part of a Rabi cycle, and reach the halfway state after a quarter-cycle. (the dwell ends, and the next one starts in the opposite phase, at the half-way point).

As we shall see, the duration of an episode is variable. The length of a dwell is the sum of the durations of all the consecutive episodes that return to the initial eigenstate, plus the final episode. 
Consider a system that is modelled by time-steps of duration $\delta t$. The probability of a measurement occurring at any step is $R \delta t$. Suppose the system starts in state $|1\rangle$, at the 'top' of the Rabi cycle. In the absence of any measurement, the density operator at time $t$ is

$$
\hat{\rho}=\cos ^{2}\left(\frac{\Omega t}{2}\right)|1\rangle\left\langle 1\left|+\sin ^{2}\left(\frac{\Omega t}{2}\right)\right| 0\right\rangle\langle 0|+i \sin \left(\frac{\Omega t}{2}\right) \cos \left(\frac{\Omega t}{2}\right)(|0\rangle\langle 1|-| 1\rangle\langle 0|) .
$$

Each time-step can have three possible outcomes:

(1) With probability $(1-R \delta t)$, no measurement takes place. Time moves forward by one step.

(2) With probability $R \delta t \cos ^{2}\left(\frac{\Omega t}{2}\right)$, a measurement takes place, and the atom returns to the state $|1\rangle$. This signals the end of this episode and the start of the next episode in the same dwell.

(3) With probability $R \delta t \sin ^{2}\left(\frac{\Omega t}{2}\right)$ this measurement switches the atom to state $|0\rangle$. This implies the end of this episode and of this dwell.

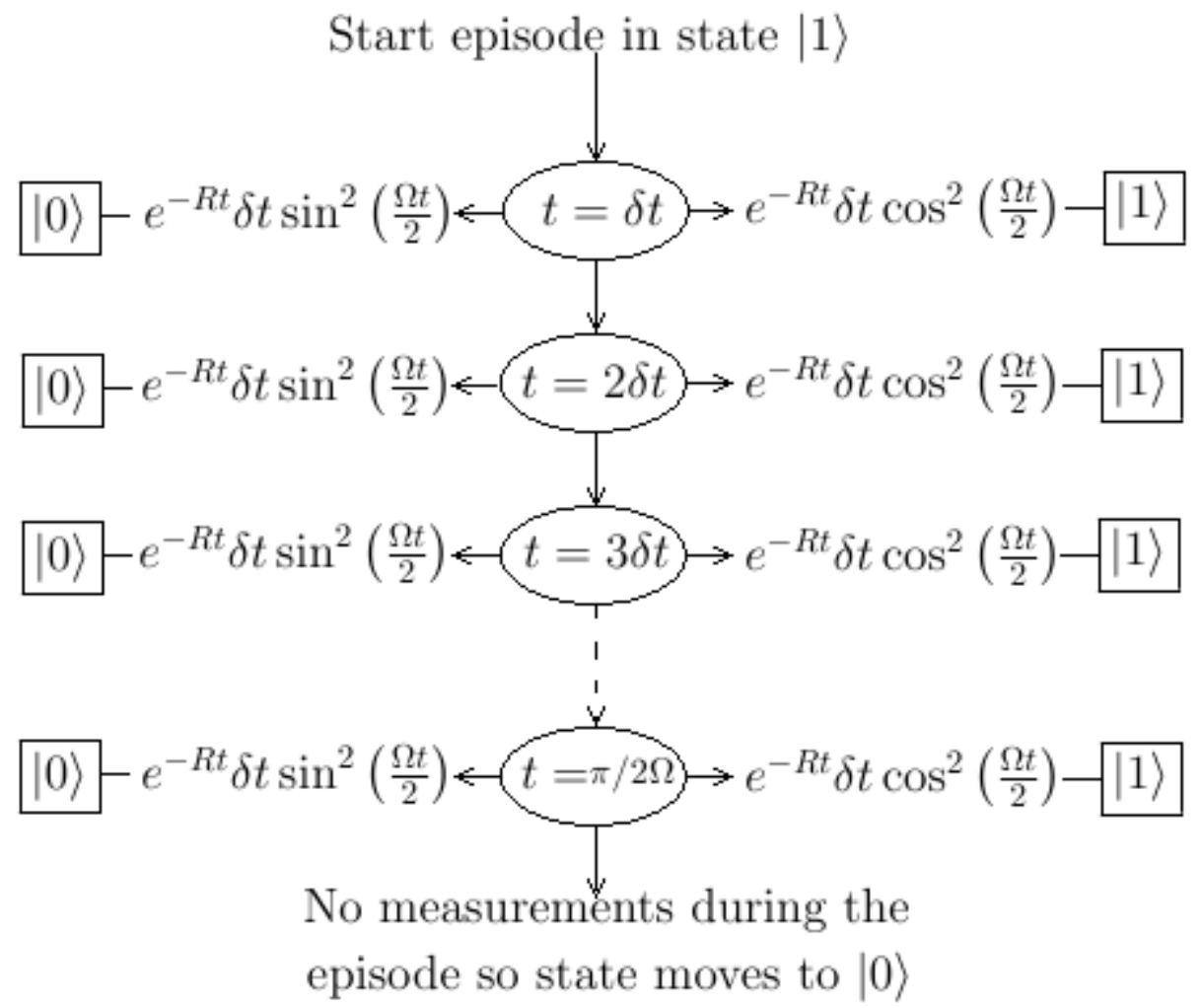

Figure 11. Probability tree showing the possible outcomes of an episode

Figure 11 is a probability tree for such an episode. The ellipses are intermediate states reached by time-steps in which no measurements take place. Rectangles are terminal nodes . Each arc is marked with the probability that will be followed.

- The probability that the atom reaches time $t$ without a measurement is $(1-R \delta t)^{t / \delta t} \approx e^{-R t}$.

- The probability that the atom returns to eigenstate $|1\rangle$ at time $t$ is $e^{-R t} \delta t \cos ^{2}\left(\frac{\Omega t}{2}\right)$.

- Similarly the probability that the atom switches to state $|0\rangle$ at time $t$ is $e^{-R t} \delta t \sin ^{2}\left(\frac{\Omega t}{2}\right)$. 
- The duration $t_{n}$ of a quarter Rabi cycle (when no measurements take place) is

$$
t_{n}=\frac{1}{4} \times \frac{2 \pi}{\Omega}=\frac{\pi}{2 \Omega} .
$$

The probability $p_{0}$ that the atom reaches the end of chain, a quarter way round the Rabi cycle, without any measurements is

$$
p_{0}=\exp \left(-\frac{R \pi}{2 \Omega}\right)
$$

The overall probability $p_{1}$ that the atom completes the episode and returns to the starting eigenstate is

$$
p_{1}=\sum_{t=0}^{t=t_{n}} \delta t R \cos ^{2}\left(\frac{\Omega t}{2}\right)
$$

Similarly, the overall probability $p_{2}$ that the atom completes the episode and switches to the opposite eigenstate is

$$
p_{2}=\sum_{t=0}^{t=t_{n}} \delta t R \sin ^{2}\left(\frac{\Omega t}{2}\right)
$$

As $\delta t \rightarrow 0$ these relations can be replaced by

$$
\begin{aligned}
p_{1} & =\int_{0}^{t_{n}} d t R \cos ^{2}\left(\frac{\Omega t}{2}\right), \\
p_{2} & =\int_{0}^{t_{n}} d t R \sin ^{2}\left(\frac{\Omega t}{2}\right) .
\end{aligned}
$$

The average length $A$ of each episode that ends with an 'up' measurement can be found by summing the probabilities of exit at each stage, multiplied by the time of exit. As this type of episode is not the only possible outcome of the evolution, we must divide by $p_{1}$, the probability of the outcome. We may put:

$$
A=\int_{0}^{t_{n}} d t t R \cos ^{2}\left(\frac{\Omega t}{2}\right) / p_{1}
$$

Similarly, the average length $B$ of an episode that ends with a 'down' measurement is

$$
B=\int_{0}^{t_{n}} d t t R \sin ^{2}\left(\frac{\Omega t}{2}\right) / p_{2}
$$

Equivalent adjustments must be made to all calculations of average episode length.

As $p_{1}$ is a probability $\left(p_{1} \leq 1\right), d_{1}$, the total length of a type 1 segment, is:

$$
d_{1}=A\left(1+p_{1}+p_{1}^{2}+p_{1}^{3}+\ldots\right)+B=A \sum_{n=0}^{\infty} p_{1}^{n}+B=\frac{A}{1-p_{1}}+B
$$


Similarly, the length of a type 2 segment is

$$
d_{2}=\frac{A}{1-p_{1}}+t_{n}
$$

The statistics of initial segments can be calculated in a similar way to those of final segments, except that:

- Each initial segment consists of only one episode

- The segment starts at the half-way state

The following equations yield the required statistics. $p_{3}$ to $p_{5}$ are probabilities, and $d_{3}$ to $d_{5}$, expected durations.

\begin{tabular}{|l|l|}
\hline$p_{3}$ (segment reaches the eigenvalue state (type 3$\left.)\right)$ & $e^{-t_{n}}$ \\
$p_{4}($ segment ends with an 'up' measurement(type 4$\left.)\right)$ & $\int_{0}^{t_{n}} d t e^{-R t} \cos ^{2}\left(\frac{\Omega t}{2}-\pi t_{n}\right)$ \\
$p_{5}($ segment ends with a 'down' measurement (type 5$\left.)\right)$ & $\int_{0}^{t_{n}} d t e^{-R t} \sin ^{2}\left(\frac{\Omega t}{2}-\pi t_{n}\right)$ \\
$d_{3}$ (duration of a type 3 segment) & 0.25 Rabi cycle $=t_{n}$ \\
$d_{4}$ (duration of a type 4 segment) & $\int_{0}^{t_{n}} d t t e^{-R t} \cos ^{2}\left(\frac{\Omega t}{2}-\pi t_{n}\right) / p_{4}$ \\
$d_{5}$ (duration of a type 5 segment) & $\int_{0}^{t_{n}} d t t e^{-R t} \sin ^{2}\left(\frac{\Omega t}{2}-\pi t_{n}\right) / p_{5}$ \\
\hline
\end{tabular}

Next we consider the average duration of dwells (as opposed to segments). There are two patterns of dwell that start at the eigenvalue, and no fewer than six that start with an initial segment. Figure 12 is a taxonomy of these different dwell types. Each arc of the graph is labelled with the probability that the arc is traversed, and each dwell type is marked with its duration.

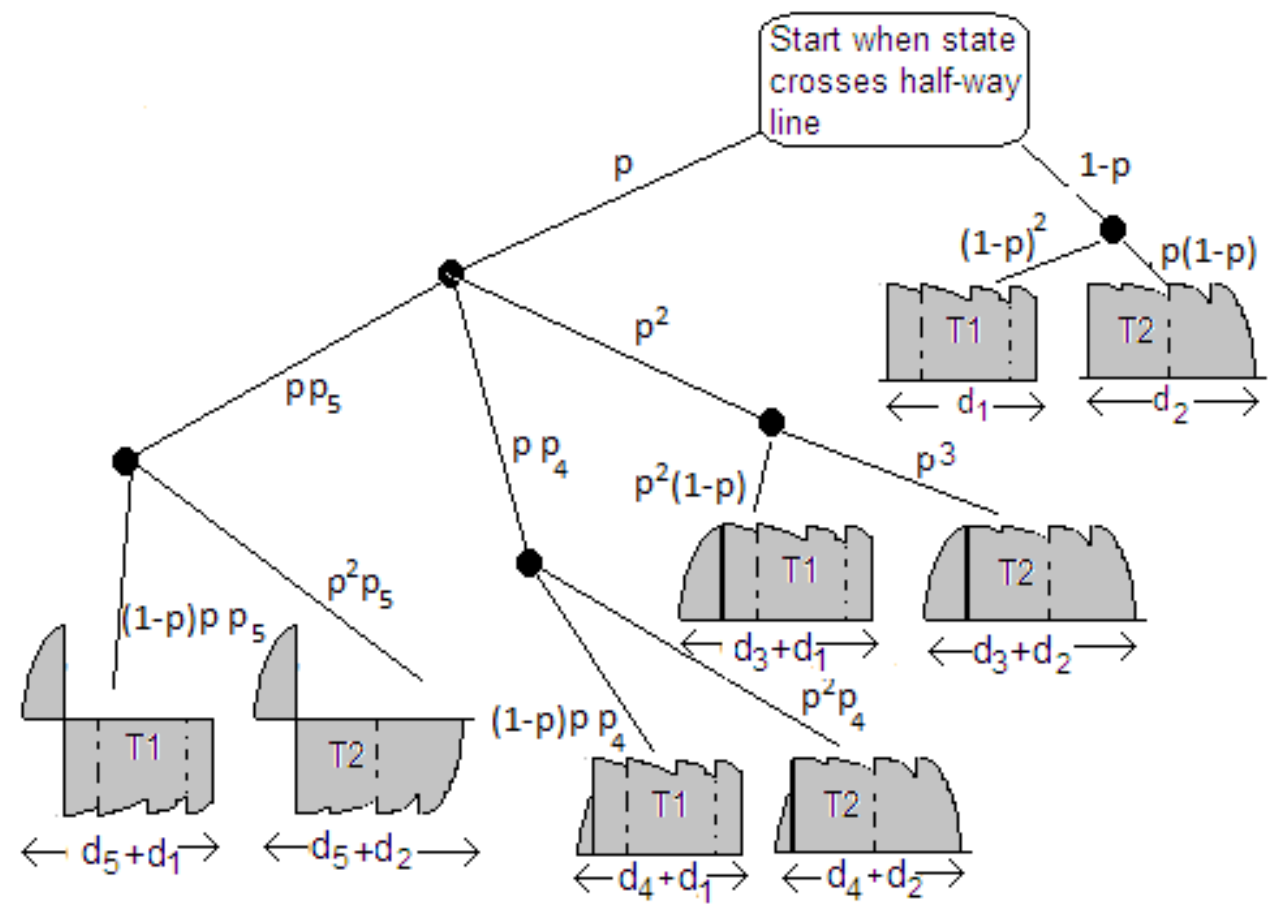

Figure 12. Taxonomy of dwell types

The average dwell length for each of many different values of $R$ was calculated by summing the durations of each of the dwell types, multiplied by the probability of their occurrence. Where 
the initial segment ends with a 'down' measurement, the calculation accounts for two dwells (one of each phase), so the contribution from this type of dwell is halved.

\subsubsection{Statistics of measurement-centred dwells}

For this part of the investigation the simulator was adjusted to detect steps in which the probability of one of the energy eigenstates switched from being reater than 0.5 to less than 0.5 . A run with a given value of $R$ took 1000 Rabi cycles or $10^{6}$ steps. This produced a number of dwells varying greatly in their length, but a roughly constant factor was the standard deviation of the lengths was close to their average. This is consistent with a negative exponential distribution, as might be expected, and is typical of a random telegraph [24]. The average dwell times delivered by the separate runs were close to one another.

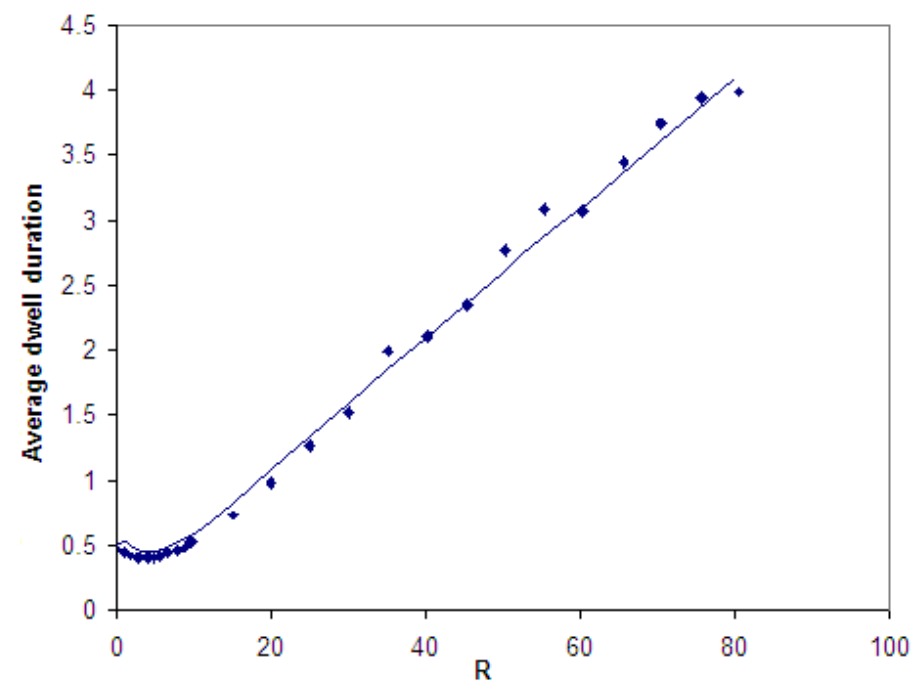

Figure 13. Measured and calculated dwell times for the state-centred method

The graph in Figure 13 shows that for $R$ values higher than 10 measurements per Rabi cycle, the relationship between $R$ and the dwell time is almost linear. In this region, the end effects due to long gaps between measurements are negligible. When measurements are frequent, most of them occur when the state of the atom is close to one of its energy eigenstates. Here the density operator is closely approximated by

$$
\hat{\rho}=\left(1-\left(\frac{\Omega t}{2}\right)^{2}\right)|1\rangle\left\langle 1\left|+\left(\frac{\Omega t}{2}\right)^{2}\right| 0\right\rangle\langle 0|+\left(\frac{i \Omega t}{2}\right)(|0\rangle\langle 1|-| 1\rangle\langle 0|)
$$

if the atom is close state $|1\rangle$, with an analogous expression if it is close to state $|0\rangle$. The probability of a quarter Rabi cycle without any measurements is negligibly small. With these approximations, the average episode duration is inversely proportional to $R$, the frequency of measurement, but the probability of an episode ending with a 'down' measurement is inversely proportional to $R^{2}$. It follows that the dwell time increases linearly with $R$.

\subsection{Observations of dynamics with error-prone measurements}

Theoretical analysis of this regime is beyond the scope of this paper, but we offer some observations derived from the simulator. 


\subsubsection{Effect of imperfect measurements on dwell times, using the state-centred method}

It is to be expected that when measurements are imperfect, any incorrect measurement is likely to force a premature change of the dwell state, and this is borne out by the simulations. We collected statistics for three measurement frequencies (in terms of measurements per Rabi cycle): $R=20, R=50$, and $R=80$.

The results are shown in figure 14, which illustrates the relationship between the error rate and the dwell time for each of the three $R$ values. Error rates in measurement were varied between 0 and 0.5. Any error rate is likely to cause the dwell time to drop sharply, in an (approximately) exponential decay.

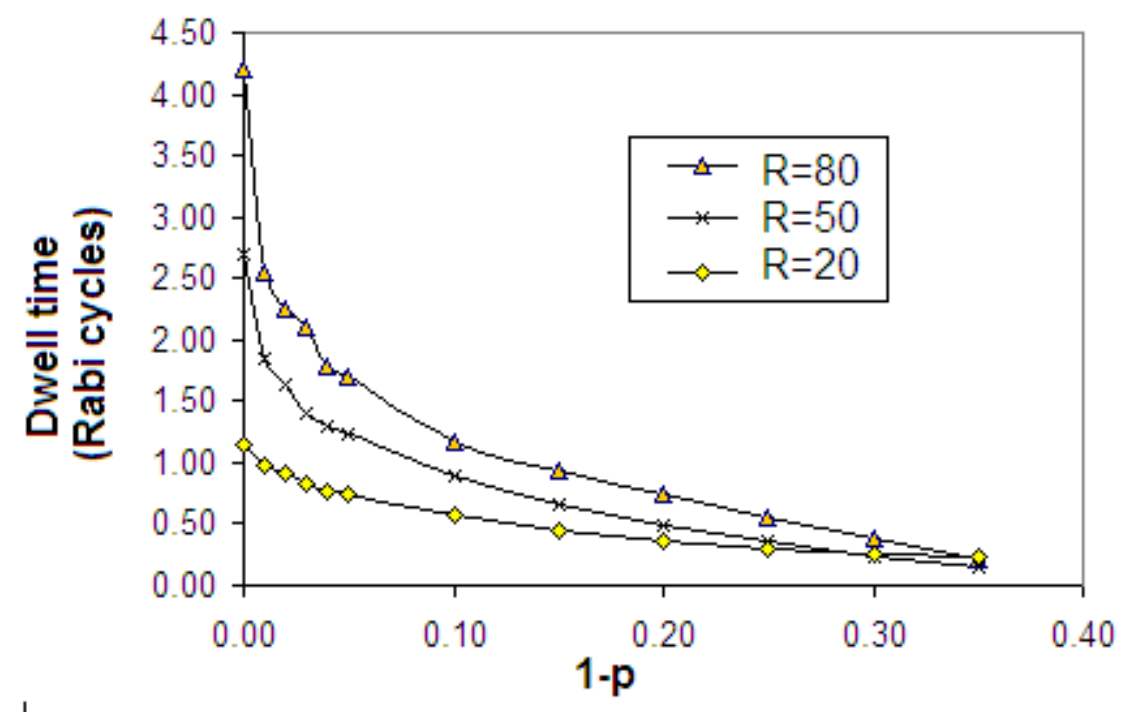

Figure 14. Relationship of dwell times and error rate in measurement

\subsubsection{Effect of imperfect measurements on the telegraphing index.}

The telegraphing indices for runs with imperfect measurements are shown in figure 15, again using three values of $R$. For $R=20$ the drop is almost linear, but for higher values of $R$, a high degree of telegraphing is maintained even for moderately high error rates.

\subsubsection{Dwell state switching under imperfect measurement}

Equation (18) shows that the state of an atom after an imperfect measurement is not in general either of the states $|1\rangle$ or $|0\rangle$, but rather a superposition of both. When $p=1$ each measurement gives an abrupt change to an eigenvalue, but when $p<1$ this is no longer the case, and switching between dwell states can take several measurements. As each measurement occurs when the atom is in an intermediate state, the outcome is indeterminate, and the state can move in either direction. This is illustrated in figure 5 .

As well as generating this trajectory, the simulator also produced the corresponding measurement record. Figure 16 is a much-magnified version of figure 5, which shows the relationship of measurements and the state of the atom. A copy of figure 5 is included for comparison.

The measurement record (alone) can also be used to find the dwell state of the atom according to the measurement-centred method. This is illustrated in figure 17. 


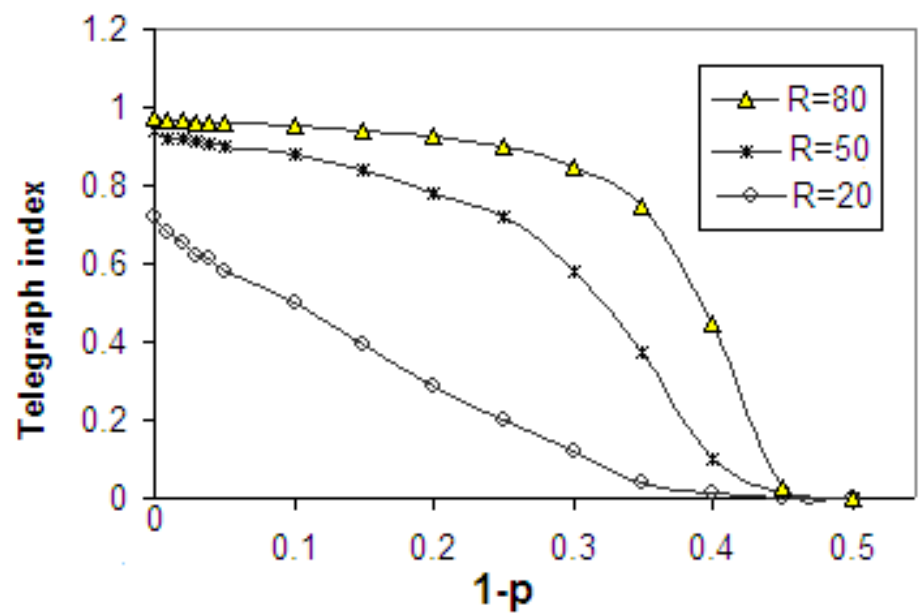

Figure 15. Relationship of telegraph index and $R$

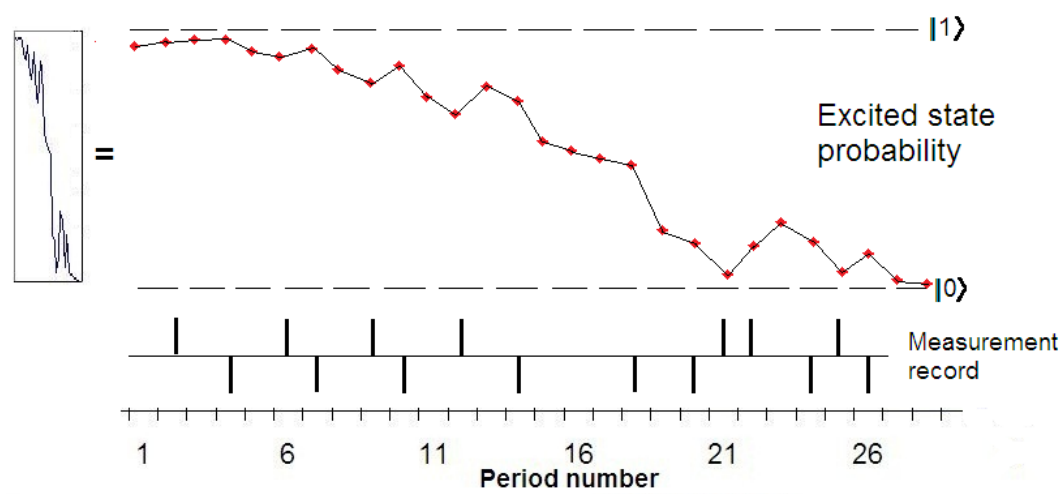

Figure 16. Imperfect measurements and the state of the atom during switching, using the state-centred method

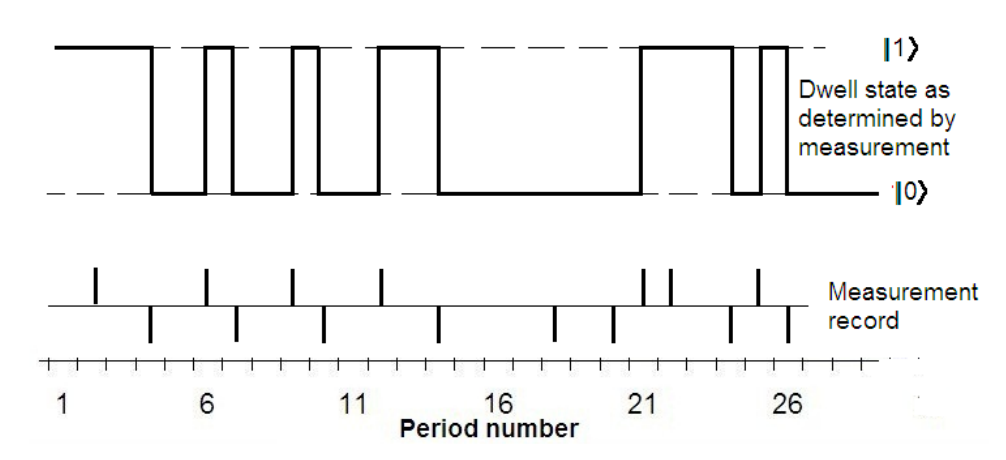

Figure 17. Dwell state of the atom during switching, using the measurement-centred method

\section{Conclusion}

The concept of frequent measurement is a useful tool for investigating the interaction of an atom with its environment. The general effect of the interaction is to exert a 'drag' on the closed evolution of the atom, so that it remains at or near one of its eigenstates longer than would

\footnotetext{
otherwise be the case. We have suggested a 'telegraph index' as a measure for this drag, and
} 
offered two possible definitions of telegraphing. Assuming perfect measurements, we have made theoretical analyses of both definitions of telegraphing. Measurements made with a simulator agree closely with those found by theoretical analyses. We found that when measurements are frequent the statistics associated with each of the definitions coincide, but when measurements are sparse the statistics diverge. The basic difference between the two definitions is that only one admits a change of the dwell state due to the uninterrupted Rabi cycle. As measurements become more frequent, the atom has an ever smaller chance of completing a quarter Rabi cycle, and the difference between the two definitions disappears.

Turning to imperfect measurements (such are defined in [1]), we have, as yet, no complete theoretical analysis, but we present graphs produced by the simulator. A general observation is that imperfect measurements make the atom much less stable; telegraphing times are reduced, the telegraph index generally drops, and switching between dwell states takes longer, with the state occupying an intermediate position in which the next measurement could easily be in either direction. During the switching process the measurement-centred model shows the dwell-state values oscillating, at a frequency similar to the frequency of measurements.

\section{Acknowledgements}

We are grateful to Jim Cresser for helpful discussions. This work was supported by the EPSRC, the Royal Society and the Wolfson Foundation.

\section{References}

[1] J.D. Cresser, S.M. Barnett, J. Jeffers and D.T.Pegg, Measurement Master Equation, Optics Communications 264, 352$361(2006)$

[2] H. Dehmelt, Laser Fluorescence Spectroscopy on Tl+ Mono-Ion Oscillator II (spontaneous quantum jumps), Bull. Am. Phys. Soc. 20, 60 (1975)

[3] R.J.Cook and H.J. Kimble, Possibility of direct observation of quantum jumps, Phys. Rev. Lett., vol. 54, pp. 1023-1026, (1985)

[4] C. Cohen-Tannoudji and J. Dalibard, Single-Atom Laser Spectroscopy. Looking for dark periods in Fluorescence Light,Europhysics Letters 1(9) pp. 441-448 (1986)

[5] P. K. Ghosh, Ion Traps, Oxford University Press, Oxford (1995)

[6] H.J.Carmichael, An Open Systems Approach to Quantum Optics, Springer, Berlin (1993)

[7] K. Mølmer, Y. Castin and J.Dalibard, Monte Carlo wave-function method in quantum optics, J.Opt. Soc. Am. B 10, $524(1993)$

[8] M.B. Plenio and P.L. Knight, The quantum-jump approach to dissipative dynamics in quantum optics, Rev. Mod. Phys. 70, 101 (1998)

[9] H.J.Carmichael, Statistical Methods in Quantum Optics 2, Springer, Berlin (2008)

[10] S.M. Barnett and P.M.Radmore, Methods in Theoretical Quantum Optics. Clarendon Press, Oxford (1997)

[11] G. Lindblad, Non-Equilibrium Entropy and Irreversibility, Delta Reidel. Dordrecht: ISBN 1-4020-0320-X (1983)

12] S. Kryszewski, J. Czechowska-Kryszk, Master equation - tutorial approach. arXiv:0801.1757v1 [quant-ph] (2008)

[13] T.M. Tong, J-L Chen, L.C. Kwek C.H. Oh, Kraus representation for density operator of arbitrary open qubit system, arXiv:quant-ph/0311091v2 (2003)

[14] B. Bellomo, S.M. Barnett, J. Jeffers, Frictional quantum decoherence, J.Phys A: Math. Theory. 40 9437-9453 (2007)

[15] S. M. Barnett and James D. Cresser, A Quantum Theory of Friction, Phys. Rev. A72 022107 (2005)

[16] J. von Neumann, Mathematical Foundations of Quantum Mechanics, Princeton University Press (1957) (English translation of the original)

[17] K. Hornburger, Monitoring approach to open quantum systems using scattering theory, EPL 77550007 (2007)

[18] M.J. Everitt, W.J. Munro and T.P. Spiller, Quantum measurement with chaotic apparatus, Phys. Lett. A 374(28) 2809 (2010)

[19] J. Salo, S. Stenholm, G. Kurizki and A.G.Kofman, The varieties of master equations, (in "Decoherence, Entanglement and Information Protection in Complex Quantum Systems"). NATP Science Series, Vol 189, Springer, (2005)

[20] M.S. Kim and P.L. Knight, Quantum-jump telegraph noise and macroscopic intensity fluctuations, Physical Review A, Vol. 36, no. 11 (1987)

[21] S.M. Barnett, Quantum Information, Oxford University Press (2009)

[22] S. Croke, S.M. Barnett, S. Stenholm, Linear transformation of quantum states, Annals of Physics 323 893-906 (2008)

[23] A.J.T.Colin, Some aspects of measurement of quantum systems, PhD thesis to be submitted to Strathclyde University, (2012)

[24] D.K.C. MacDonald, Noise and fluctuations: an introduction, John Wiley \& Sons, Inc (1962) 